\title{
Existence of solitary travelling waves in interfacial electrohydrodynamics
}

\author{
P.W. Hammerton \\ School of Mathematics, University of East Anglia, Norwich NR4 7TJ, U.K.
}

\begin{abstract}
The propagation of waves on the surface of a fluid layer of finite depth is considered in the presence of a normal electric field, due to parallel electrodes at arbitrary separation distance. The combined effect of electric field, gravity and surface tension is considered in the long-wavelength small-amplitude limit. Travelling wave solutions are characterised in terms of the Froude number, an electric Weber number and a Bond number and conditions for the existence of solitary waves are determined in terms of these parameters.
\end{abstract}

Keywords:

\section{Introduction}

The effect of normal electric fields on the occurence of solitary surface waves has been considered in a series of papers, both analytic and numeric. Two-dimensional disturbances on a horizontal sheet of fluid, have been investigated in the presence of an applied electric field in a horizontal direction $[1,2]$. A normal electric field has been considered $[3,4,5,6]$ when the field is due to parallel electrodes with separation distance comparable to the depth of the fluid. When the normal electric field tends to a constant far from the surface, corresponding to parallel electrodes with very large separation distance, a Kortweg-deVries Benjamin-Ono type equation is obtained for particular wavelength and amplitude scalings $[7,8]$. In the present paper a more general analysis for the normal electric field case derivation is provided, which allows for a wider range of wavelength and amplitude scalings and includes the effect of electrode separation distance. Conditions for the existence of travelling solitary wave solutions are determined, and numerical results for a range of parameter values are presented. 
For a horizontal fluid layer of depth $h$, small amplitude disturbances with wavelength much greater than $h$ propagate at speed $\sqrt{g h}$. For a particular scaling between disturbance amplitude and wavelength, a weakly nonlinear analysis, taking account of surface tension gives rise to the Kortweg-de-Vries equation [9], with the familiar sech ${ }^{2}$ solitary solutions. Capillary effects are characterised by the inverse Bond number $\tau=\sigma / \rho g h^{2}$ but when that is close in value to $\frac{1}{3}$ wavelength shortening means that higher order terms in the weakly nonlinear analysis must be included. A different scaling between amplitude and wavelength then gives rise to an additional fifth derivative term [9]. The inclusion of an electric field modifies these governing equations.

In the present paper we consider two horizontal parallel plates separated by distance $h+d$ with a layer of inviscid fluid, undisturbed depth $h$, lying on the lower impermeable plate. Between the fluid layer and the upper plate, a second fluid with different electric properties is present and this fluid is taken to be hydrodynamically passive. A potential difference $V_{0}$ is then applied between the two plates. Previous analysis of this problem has focused either on the case when the lower fluid is a perfect conductor, or on the case when both fluids are perfect dielectrics. In the first case the tangential component of electric field at the interface is zero, in the second case the surface charge density at the interface is zero. In both cases the tangential force at the interface due to the electric field is zero. If instead the two fluids are modelled as leaky dielectrics [10], then the interfacial tangential force is non-zero and a large-Reynolds number analysis is required, involving a hydrodynamic boundary layer below the interface. This is beyond the scope of the present paper. Here we consider the case when the lower fluid is assumed to be a perfect conductor, but the analysis can be readily modified to the case of two perfect dielectrics. In either case, a potential field $V$ is set up in the upper fluid, but when the lower fluid is taken to be a perfect conductor the potential in this region is a constant.

As well as considering the case of parallel electrodes, we also consider separately the case when the electric field tends to a constant magnitude in the vertical direction, far from the the fluid layer. We consider this case to allow direct comparison with earlier works $[7,8]$. However this case should correspond to the limit $d \rightarrow \infty$, the exact nature of the limit emerges as part of the analysis presented in $\S 3$.

The structure of the paper is as follows. In $\S 2$ the governing equation is derived in a fashion similar to previous analyses [7, 8], but without assuming any particular scaling between wavelength and amplitude, and allowing a 
more general electrical forcing. In $\S 3$ the dependence of the electric field on the electrode separation distance is considered, along with the resulting effect on the surface waves. These results are compared with other approaches $[5,8]$ and previous results then emerge as limits of this governing equation. In $\S 4$ travelling wave solutions are considered. The question of whether true solitary wave solutions are possible for particular parameter values is studied by considering when the linearised equation permits non-decaying oscillatory solutions. In $\S 5$ these predictions are compared with numerical solutions obtained using spectral methods.

\section{Governing Equation}

An impermeable electrode is located at $y_{*}=-h$ and an inviscid fluid layer occupies the region $-h<y_{*}<\eta^{*}\left(x_{*}\right)$ above the electrode, where we take $x_{*}, y_{*}$ to be the horizontal and vertical dimensional coordinates. The flow in the perturbed fluid layer is taken to be irrotational and so the velocity field is given by $\mathbf{u}^{*}=\nabla^{*} \phi^{*}$ where $\phi^{*}\left(x_{*}, y_{*}\right)$ is the dimensional velocity potential and satisfies Laplace's equation. The electrode is maintained at potential $V^{*}=0$ and we consider the case when the fluid is a perfect conductor and hence the electric field in the fluid layer is zero. Above this layer we have a dielectric medium assumed to be hydrodynamically passive, but which supports an electric field $\mathbf{E}^{*}=\nabla V^{*}$, where $V^{*}\left(x_{*}, y_{*}\right)$, the dimensional electric potential, satisfies Laplace's equation.

As previously noted we consider two different imposed electric fields. In case 1 we take the upper medium to be unbounded, with electric field $\mathbf{E}^{*} \rightarrow$ $E_{0} \mathbf{y}$ as $y^{*} \rightarrow \infty$. In case 2 we consider a second horizontal electrode located at $y_{*}=d$ and maintained at potential $V^{*}=V_{0}$. In this case $V^{*}$ satisfies Laplace's equation in the region $\eta^{*}\left(x_{*}\right)<y_{*}<d$. The set-up is illustrated in figure 1.

At the interface the fluid velocity satisfies the kinematic condition, and since the lower fluid is a perfect conductor the tangential component of the electric field is zero. The normal component of the electric field at the interface gives rise to a normal stress on the surface, which together with the effect of surface tension lead to additional terms in the Bernoulli condition at the interface.

The set of equations governing the fluid flow, the electric field and the interface conditions is non-dimensionalised using the undisturbed depth $h$, the gravitational acceleration $g$ and the magnitude of the electric field in the 
(a)

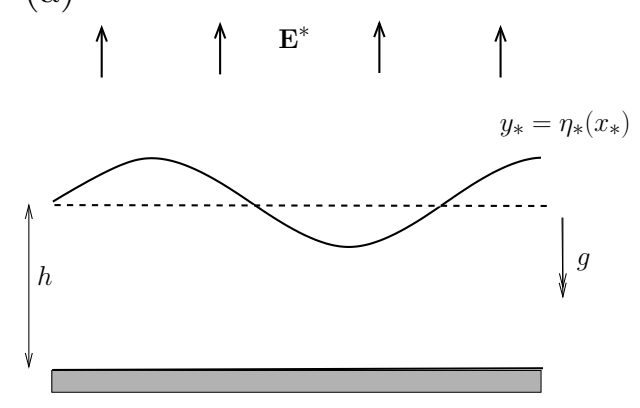

(b)

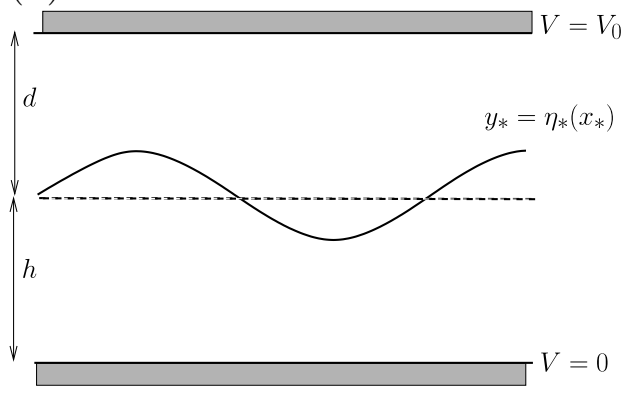

Figure 1: Fluid layer occupying the region $-h<y_{*}<\eta^{*}\left(x_{*}\right)$ with: (a) unbounded upper medium with electric field $\mathbf{E}^{*} \rightarrow \mathbf{E}^{*} \mathbf{y}$ as $y_{*} \rightarrow \infty$ (Case 1); (b) upper electrode located at $y^{*}=d$ and maintained at potential $V_{0}$ (Case 2)

upper medium $E^{*}$. For the first case considered where the upper medium is unbounded and $\mathbf{E}^{*} \rightarrow E_{0} \mathbf{y}$ as $y^{*} \rightarrow \infty$, we take $E^{*}=E_{0}$. For the second case we take $E^{*}=V_{0} / d$, where $V_{0}$ is the potential of the upper electrode. Now, changing to a frame moving with the shallow water linear wave speed $\sqrt{g h}$, and assuming that the amplitude of the perturbation of the surface is $O(\delta h)$ and that the wavelength is $O\left(\epsilon^{-1} h\right)$, we write

$$
\eta=\frac{1}{\delta h} \eta^{*}, \quad x=\frac{\epsilon}{h}\left(x_{*}-\sqrt{g h} t_{*}\right), \quad y=\frac{1}{h} y_{*} .
$$

In the small-amplitude long-wavelength limit $(\delta, \epsilon \ll 1)$, specific scalings between $\delta$ and $\epsilon$ are considered elsewhere [8], but here we describe a general method without specifying a particular scaling between the two small parameters, and clarifying the required scalings of the other quantities.

At this stage we choose arbitrary scalings for the remaining variables

$$
t=K_{1} \sqrt{\frac{g}{h}} t_{*}, \quad \phi=\frac{K_{2}}{h \sqrt{g h}} \phi^{*}, \quad V=\frac{K_{3}}{h E^{*}} V^{*}, \quad Y=\frac{K_{3}}{h} y_{*},
$$

where $Y$ is the vertical coordinate in upper fluid, chosen so that $V_{Y}$, the vertical component of the non-dimensional electric field is $O(1)$. With this choice of rescaling the interface is given by $y=\delta \eta$ and the governing equation for the lower fluid flow becomes

$$
\epsilon^{2} \phi_{x x}+\phi_{y y}=0 \quad-1<y<\delta \eta
$$


with the no-slip condition $\phi_{y}=0$ on $y=-1$ and the kinematic condition at the interface

$$
\delta K_{1} K_{2} \eta_{t}-\delta \epsilon K_{2} \eta_{x}+\epsilon^{2} \delta \phi_{x} \eta_{x}-\phi_{y}=0, \quad y=\delta \eta .
$$

In the upper medium, the electric potential field $V$ satisfies

$$
V_{x x}+\left(\frac{K_{3}}{\epsilon}\right)^{2} V_{Y Y}=0 \quad Y>K_{3} \delta \eta
$$

and the scaling factor for the electric field is chosen as $K_{3}=\epsilon$ so that $V$ satisfies Laplace's equation. The condition that the tangential component of electric field at the interface is zero then becomes

$$
V_{x}+\delta \epsilon \eta_{x} V_{Y}=0, \quad Y=\epsilon \delta \eta,
$$

while the upper boundary condition depends on whether we are considering an unbounded upper medium or the case of an upper electrode,

$$
V_{Y} \rightarrow 1 \quad \text { as } Y \rightarrow \infty \quad(\text { Case } 1) ; \quad V\left(Y=\frac{\epsilon d}{h}\right)=\frac{\epsilon d}{h} \quad \text { (Case 2) }
$$

Finally the hydrodynamic flow and the electric field are linked through Bernoulli's equation at the interface which takes the form

$$
\delta R+\frac{E_{b}}{S} Q-\frac{\epsilon^{2} \delta \tau}{S^{\frac{3}{2}}} \eta_{x x}=\text { constant, } \quad \text { on } \quad y=\delta \eta .
$$

where the non-dimensional parameters characterising the effects of the electric field and the surface tension are

$$
E_{b}=\frac{\varepsilon E^{* 2}}{\rho g h}, \quad \tau=\frac{\sigma}{\rho g h^{2}},
$$

an electric Weber number and an inverse Bond number respectively. Here $\sigma$ is the surface tension parameter and $\varepsilon$ is the dielectric constant of the upper medium. The terms $R, Q, S$ are given by

$$
\begin{aligned}
R & =\frac{K_{1}}{\delta K_{2}} \phi_{t}-\frac{\epsilon}{\delta K_{2}} \phi_{x}+\frac{1}{2 \delta K_{2}^{2}}\left(\epsilon^{2} \phi_{x}^{2}+\phi_{y}^{2}\right)+\eta \\
Q & =-\frac{1}{2}\left(V_{Y}^{2}-V_{x}^{2}\right)\left(1-\delta^{2} \epsilon^{2} \eta_{x}^{2}\right)+2 \delta \epsilon \eta_{x} V_{x} V_{Y}, \\
S & =1+\delta^{2} \epsilon^{2} \eta_{x}^{2} .
\end{aligned}
$$


In the subsequent analysis we consider $E_{b}$ and $\tau$ to be $O(1)$ or smaller.

At this stage we choose distinguished scalings motivated by physical balances. The scaling $K_{2}$ is chosen so that the leading order balance in Bernoulli's equation is between $\eta$ and $\phi_{x}$, and $K_{1}$ so that the time derivatives and quadratic nonlinearity enter at the same order. This choice of balances gives

$$
K_{1}=\delta \epsilon, \quad K_{2}=\frac{\epsilon}{\delta} .
$$

hence the governing equations for the velocity potential and the electric potential in the lower and upper fluids respectively are given by

$$
\begin{array}{rlrl}
\epsilon^{2} \phi_{x x}+\phi_{y y} & =0, & & -1<y<\delta \eta \\
V_{x x}+V_{Y Y} & =0, & Y>\epsilon \delta \eta .
\end{array}
$$

The conditions at the interface then become,

$$
\begin{aligned}
\delta \epsilon^{2}\left(\eta_{t}+\phi_{x} \eta_{x}\right)-\epsilon^{2} \eta_{x}-\phi_{y} & =0, \quad y=\delta \eta, \\
V_{x}+\delta \epsilon \eta_{x} V_{Y} & =0, \quad Y=\epsilon \delta \eta, \\
\delta R+E_{b} Q-\epsilon^{2} \delta \tau \eta_{x x}+O\left(\delta^{2} \epsilon^{2}\right) & =\text { constant, } \quad y=\delta \eta,
\end{aligned}
$$

where

$$
\begin{aligned}
R & =\eta-\phi_{x}+\delta\left(\phi_{t}+\frac{1}{2} \phi_{x}^{2}\right)+\frac{\delta}{2 \epsilon^{2}} \phi_{y}^{2} \\
Q & =-\frac{1}{2}\left(V_{Y}^{2}-V_{x}^{2}\right)+2 \delta \epsilon \eta_{x} V_{x} V_{Y} .
\end{aligned}
$$

We begin by considering the term involving the electric field. Motivated by the boundary conditions on $V$, we set

$$
V=Y+\delta \epsilon W
$$

where $W(x, Y)$ satisfies Laplace's equation, $W_{x}=-\eta_{x}+O(\delta \epsilon)$ at the interface and from (1)

$$
\left.W_{Y} \rightarrow 0 \quad \text { as } Y \rightarrow \infty \quad(\text { Case } 1) ; \quad W\left(Y=\frac{\epsilon d}{h}\right)=0 \quad \text { (Case } 2\right) .
$$

In terms of the new function $W$ the contribution to (7) from the electric field is given by $Q=-\frac{1}{2}-\delta \epsilon W_{Y}+O\left((\delta \epsilon)^{2}\right)$, where $W_{Y}$ is evaluated at the interface. We return to the calculation of the electric field in $\S 3$ but at this 
stage we retain as much generality as possible and write $W_{Y}$ at the interface as $\mathcal{P}[\eta]$, where this symbolic dependence on the interface position, $\eta$, depends on the boundary conditions on the electric field in the upper medium. Hence

$$
Q=-\frac{1}{2}-\delta \epsilon \mathcal{P}[\eta]+O\left((\delta \epsilon)^{2}\right)
$$

and the equations relating $\eta(x)$ and $\phi(x, y)$ on $y=\delta \eta$ become

$$
\begin{aligned}
\phi_{y} & =-\epsilon^{2} \eta_{x}+\epsilon^{2} \delta\left(\eta_{t}+\phi_{x} \eta_{x}\right), \\
\eta & =\phi_{x}-\delta\left(\phi_{t}+\frac{1}{2} \phi_{x}^{2}\right)+E_{b} \epsilon \mathcal{P}[\eta]+\epsilon^{2} \tau \eta_{x x}+O\left(\delta \epsilon^{2} E_{b}\right) .
\end{aligned}
$$

Rearranging (9) by repeated substitution of $\eta$ into the right hand side gives $\eta$ in terms of $\phi$,

$$
\begin{aligned}
\eta=\phi_{x}- & \delta\left(\phi_{t}+\frac{1}{2} \phi_{x}^{2}\right)+E_{b} \epsilon \mathcal{P}\left[\phi_{x}\right]+\epsilon^{2} \tau \phi_{x x x}+\epsilon^{4} \tau^{2} \phi_{x x x x x} \\
+ & O\left(\epsilon^{6}, \epsilon \delta E_{b}, \epsilon^{3} E_{b}, \epsilon^{2} E_{b}^{2}\right) .
\end{aligned}
$$

In an analysis of this form, care must be taken to consider all terms which may enter the final model equation at leading order. There may appear to be an inconsistency in the terms retained in the expression for $\eta$ above, in that the $O\left(\epsilon^{4}\right)$ has been retained. The reason for this becomes clear later in the exposition.

Next $\eta$ can be eliminated from (8) to give

$$
\begin{array}{r}
\phi_{y}=-\epsilon^{2} \phi_{x x}+2 \epsilon^{2} \delta\left(\phi_{x t}+\phi_{x} \phi_{x x}\right)-\epsilon^{3} E_{b} \mathcal{P}_{x}\left[\phi_{x}\right]-\epsilon^{4} \tau \phi_{x x x x} \\
-\epsilon^{6} \tau^{2} \phi_{x x x x x x}+O\left(\delta \epsilon^{4}, \epsilon^{8} \tau^{3}, \epsilon^{3} \delta E_{b}, E_{b} \epsilon^{5}, \epsilon^{4} E_{b}^{2}, \delta^{2} \epsilon^{2}\right),
\end{array}
$$

on the boundary $y=\delta \eta$. Finally transferring the boundary conditions from $y=\delta \eta$ to $y=0$ we have

$$
\phi_{y}(\delta \eta)=\phi_{y}(0)+\delta \eta \phi_{y y}(0)+O\left(\delta^{2}\right)=\phi_{y}-\epsilon^{2} \delta \eta \phi_{x x}+O\left(\epsilon^{2} \delta^{2}\right)
$$

from which it follows that the set of equations to be solved for $\phi(x, t)$ becomes,

$$
\begin{array}{rc}
\phi_{y y}=-\epsilon^{2} \phi_{x x}, & -1<y<0 \\
\phi_{y}=0, & y=-1 \\
\phi_{y}=-\epsilon^{2} \phi_{x x}+\epsilon^{2} \delta\left(2 \phi_{x t}+3 \phi_{x} \phi_{x x}\right)-\epsilon^{3} E_{b} \mathcal{P}_{x}\left[\phi_{x}\right] & y=0 . \\
& \quad-\epsilon^{4} \tau \phi_{x x x x}-\epsilon^{6} \tau^{2} \phi_{x x x x x x} \\
& +O\left(\delta \epsilon^{4}, \epsilon^{8}, \epsilon^{3} \delta E_{b}, E_{b} \epsilon^{5}, \epsilon^{4} E_{b}^{2}, \delta^{2} \epsilon^{2}\right)
\end{array}
$$


Solving the first of these equations subject to the boundary condition at $y=-1$ gives

$$
\phi=G-\frac{1}{2}(y+1)^{2} G^{(i i)} \epsilon^{2}+\frac{1}{4 !}(y+1)^{4} G^{(i v)} \epsilon^{4}+-\frac{1}{6 !}(y+1)^{6} G^{(v i)} \epsilon^{6}+O\left(\epsilon^{8}\right),
$$

where $G(x, t)$ is an as yet undetermined function. Note that terms such as $\epsilon^{2} H_{1}(x, t)$ and $\delta H_{2}(x, t)$ can be included in this expansion, but that with suitable normalisation, such terms can then be incorporated into the leading term $G(x, t)$. Substituting into the boundary condition at $y=0$ finally gives,

$$
\begin{aligned}
& \epsilon^{2} \delta\left(2 G_{x t}+3 G_{x} G_{x x}\right)-\epsilon^{3} E_{b} \mathcal{P}_{x}\left[G_{x}\right]-\epsilon^{4}\left(\tau-\frac{1}{3}\right) G^{(i v)} \\
& \quad+\epsilon^{6}\left(\frac{1}{2} \tau-\tau^{2}-\frac{1}{30}\right) G^{(v i)}+O\left(\delta \epsilon^{4}, \epsilon^{8}, \epsilon^{3} \delta E_{b}, E_{b} \epsilon^{5}, \epsilon^{4} E_{b}^{2}, \delta^{2} \epsilon^{2}\right)=0
\end{aligned}
$$

and hence, dropping the smaller correction terms,

$$
2 \eta_{t}+3 \eta \eta_{x}=\frac{\epsilon^{2}\left(\tau-\frac{1}{3}\right)}{\delta} \eta_{x x x}+\frac{\epsilon E_{b}}{\delta} \mathcal{P}_{x}[\eta]-\frac{1}{45} \frac{\epsilon^{4}}{\delta} \eta_{x x x x x}
$$

since $G_{x}=\eta$ to leading order. It is now clear why the $O\left(\epsilon^{4}\right)$ terms were retained along with the $O\left(\epsilon^{2}\right)$ terms, since when $\tau$ is close to the critical value of $\frac{1}{3}$ then this apparently smaller term can enter at leading order.

The importance of the electric field and surface tension terms is determined by the relative size of the coefficients involving $\delta$, the non-dimensional wave disturbance amplitude, $\epsilon^{-1}$ the non-dimensional wavelength, $\tau$ the nondimensional surface tension parameter and $E_{b}$ the non-dimensional electric field parameter. The derivation of (11) is based on both $\delta$ and $\epsilon$ being small, but without assuming anything about their relative sizes, while as previusly noted, $E_{b}$ and $\tau$ are $O(1)$ or smaller. Taking $E_{b}$ and $\tau$ large requires more careful analysis of the higher order terms dropped in the asymptotic analysis and is not considered in the present paper.

We now consider the leading order balance for different relative sizes of $\delta, \epsilon, E_{b}$ and $\widehat{\tau}=\tau-\frac{1}{3}$. If we first consider the case

$$
\delta \gg \max \left(\epsilon E_{b}, \epsilon^{2} \widehat{\tau}, \epsilon^{4}\right),
$$

then the leading order equation exhibits nonlinear steepening and hence a shortening lengthscale before the terms on the right hand side of (11) become comparable to the nonlinear term. The shortening lengthscale is equivalent to 
(a)

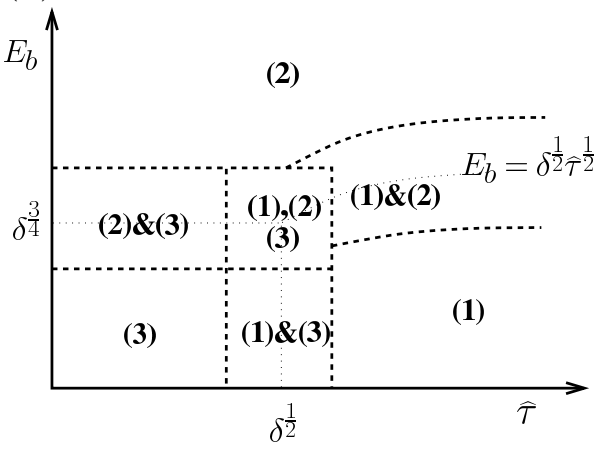

(b)

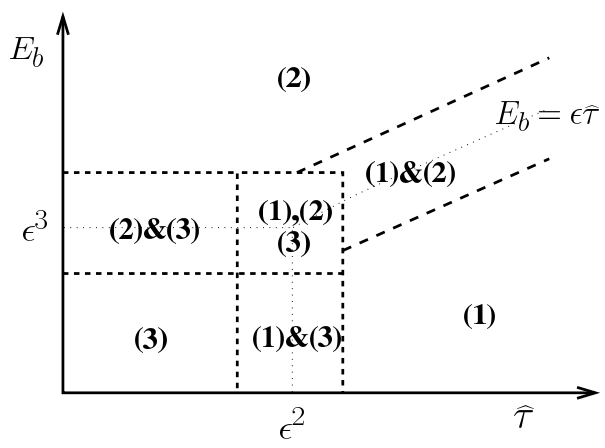

Figure 2: Schematic diagram showing the dominant terms on the right hand side of (11) as a function of $\widehat{\tau}$ and $E_{b}$ in terms of (a) disturbance amplitude, $\delta$, and (b) wavelength, $\epsilon^{-1}$.

an increase in $\epsilon$; which term becomes comparable to the nonlinearity depends on the size of the parameters $E_{b}$ and $\widehat{\tau}$.

Unless $\tau$ is close to $\frac{1}{3}$, the third derivative term in (11) is larger than the fifth derivative term and so the effect of surface tension and the electric field enter at the same order as the quadratic nonlinearity when

$$
\epsilon=\delta^{\frac{1}{2}}, \quad E_{b}=O\left(\delta^{\frac{1}{2}}\right) .
$$

If $\tau-\frac{1}{3}$ is small then all three terms on the right hand side of (11) enter at the same order as the nonlinearity if

$$
\tau-\frac{1}{3}=O\left(\delta^{\frac{1}{2}}\right), \quad \epsilon=\delta^{\frac{1}{4}}, \quad E_{b}=O\left(\delta^{\frac{3}{4}}\right) .
$$

The structure of the governing equation for different ranges of the parameter is summarised in figure 2, and this result illustrates the power of the present method, rather than specifying the key scalings at the start of the asymptotic analysis.

Before we consider the form of solutions in the different regimes we must determine the electric forcing term for the two cases of electric field previously discussed.

\section{Electric Field}

In order to calculate the term in the governing equation for the surface elevation, we must solve for the electric field in the upper medium. Linearis- 
ing so the boundary condition on the interface is applied at $Y=0$, at leading order we have

$$
W_{x x}+W_{Y Y}=0, \quad W_{x}(x, 0)=-\eta_{x} \quad\left\{\begin{array}{lll}
W_{Y} \rightarrow 0 & Y \rightarrow \infty & \text { (Case 1), } \\
W=0 & Y=\frac{\epsilon d}{h} & \text { (Case 2), }
\end{array}\right.
$$

and the effect of the electric field on the surface wave is given by $\mathcal{P}[\eta]=$ $W_{Y}(x, 0)$. We now determine $\mathcal{P}[\eta]$ for the two cases of imposed electric field.

\subsection{Case 1 - Unbounded upper medium}

In case 1 , since $W$ satisfies Laplace's equation on $Y>0$ and $W_{Y}$ decays as $Y \rightarrow \infty$, it can be readily shown that the first partial derivatives throughout $Y>0$ are related by

$$
W_{Y}=-\mathcal{H}\left[W_{x}\right], \quad W_{x}=\mathcal{H}\left[W_{Y}\right] .
$$

Here $\mathcal{H}$ denotes the Hilbert transform with respect to $x$, defined [11] as the Cauchy principal value of a convolution integral,

$$
\mathcal{H}[f]=P V\left(\frac{1}{\pi} \int_{-\infty}^{\infty} \frac{f(s, t)}{x-s} d s\right) .
$$

From this definition it immediately follows that,

$$
\frac{d}{d x}(\mathcal{H}(f))=\mathcal{H}\left(f_{x}\right), \quad \mathcal{H}\left[e^{\mathrm{i} k x}\right]=\frac{|k|}{\mathrm{i} k} e^{\mathrm{i} k x} .
$$

At the interface, $W_{x}=-\eta_{x}$ at leading order, so $W_{Y}=\mathcal{H}\left[\eta_{x}\right]$ and hence

$$
\mathcal{P}[\eta]=\mathcal{H}\left[\eta_{x}\right]
$$

Thus for an unbounded upper medium the governing equation for surface elevation becomes

$$
2 n_{t}+3 n n_{x}=\frac{\epsilon^{2}\left(\tau-\frac{1}{3}\right)}{\delta} n_{x x x}+\frac{\epsilon E_{b}}{\delta} \mathcal{H}\left[n_{x x}\right]-\frac{1}{45} \frac{\epsilon^{4}}{\delta} n_{x x x x x} .
$$

For the two specific scalings (12) and (13) previously discussed, the resulting governing equation corresponds to equation (29) and (40) respectively of Gleeson et al [8], allowing for the alternative sign convention of the Hilbert transform. 


\subsection{Case 2 - Parallel Electrodes}

Writing $D=\epsilon d / h$ we consider the problem,

$$
W_{x x}+W_{Y Y}=0, \quad W(x, 0)=-\eta(x), \quad W(x, D)=0 .
$$

Note that we could add an arbitrary constant to $W(x, 0)$ though it can be readily shown that this will have no effect on the final solution.

By taking Fourier transforms with respect to $x, W(x, Y)$ can be obtained in the form of a convolution integral

$$
\begin{aligned}
W(x, Y) & =-\int_{-\infty}^{\infty} \eta(x-s) g(s, Y) d s, \\
g(x, Y) & =\frac{1}{2 \pi} \int_{-\infty}^{\infty} \frac{\sinh (k(D-Y))}{\sinh (k D)} e^{\mathrm{i} k x} d k
\end{aligned}
$$

and so $\mathcal{P}[\eta]=W_{Y}(x, 0)$ is given by

$$
\begin{aligned}
\mathcal{P}[\eta] & =\lim _{Y \rightarrow 0}\left(\int_{-\infty}^{\infty} \eta(x-s) h(s, Y) d s\right), \\
h(x, Y) & =\frac{1}{2 \pi} \int_{-\infty}^{\infty} \frac{k \cosh (k(D-Y))}{\sinh (k D)} e^{\mathrm{i} k x} d k
\end{aligned}
$$

It should be noted that while the integral for $h(x, Y)$ does not converge for $Y=0$, it does for all $Y>0$ since

$$
\frac{k \cosh (k(H-Y))}{\sinh (k H)} \rightarrow|k| \exp (-|k| Y), \quad|k| \rightarrow \infty .
$$

Hence $\mathcal{P}[\eta]$ which appears in (11) has been determined for general $D$.

\subsection{Comparison with other work}

The effect of the electric field on the wave equation is through the term $\mathcal{P}_{x}[\eta]$ with the form of $\mathcal{P}$ given by (16) and (18) for the two cases considered. The governing PDE (11) is best solved numerically using a pseudospectral scheme [12] in which all linear terms involving spatial derivatives, including the electric forcing term, are evaluated in Fourier space, but the solution is advanced forwards in time in physical space. Also, in $\S 4$ travelling waveforms 
are calculated in spectral space. Hence we require $\mathcal{P}[\eta]$ when $\eta(x)=e^{\mathrm{i} k x}$, and obtain

$$
\mathcal{P}\left[e^{\mathrm{i} k x}\right]=f(k) e^{\mathrm{i} k x}, \quad f(k)= \begin{cases}|k| & \text { Case 1, } \\ |k| \operatorname{coth}(|k| D) & \text { Case 2. }\end{cases}
$$

The result for case 1 follows from the definition of the Hilbert transform, while the result for case 2 follows immediately from the solution in Fourier transform space. It is then clear that case 1 is the limit of case 2 as $D \rightarrow \infty$. More precisely, since $D=\epsilon d / h$, the case of an unbounded upper medium previously studied $[7,8]$ corresponds to parallel electrodes with separation large compared with the disturbance wavelength $\epsilon^{-1} h$.

If $d$ is small compared with the disturbance wavelength (that is $D \ll 1$ ) then

$$
f(k)=\frac{1}{D}\left(1+\frac{1}{3} k^{2} D^{2}+O\left(D^{4}\right)\right), \quad \Longrightarrow \quad \mathcal{P}[\eta]=\frac{1}{D} \eta-\frac{D}{3} \eta_{x x}+O\left(D^{3}\right),
$$

and hence from (11),

$$
2\left(\eta_{t}-c \eta_{x}\right)+3 \eta \eta_{x}=\frac{\epsilon^{2}}{\delta}\left(\tau-\frac{1}{3}-\frac{E_{b} d}{3 h}\right) \eta_{x x x}-\frac{1}{45} \frac{\epsilon^{4}}{\delta} \eta^{(v)}, \quad c=\frac{E_{b} h}{2 \delta d} .
$$

So the effect of the electric field is to shift the wave speed and modify the coefficient of the dispersion term. Thus the critical value for $\tau_{c}$ for the inverse Bond number when the fifth derivative term becomes significant is now

$$
\tau_{c}=\frac{1}{3}+\frac{E_{b} d}{3 h} .
$$

This can be compared to the results of $[4,5]$, where it was assumed that the electrode separation and the depth of the fluid layer are comparable. In this case care must be taken in interpreting (11), since $\mathcal{P}_{x}[\eta]$ is now large and terms previously dropped as being uniformly small in the derivation of (11) may become significant. However, if the electrode separation is such that $\epsilon^{-1} \gg d / h \gg 1$, with $E_{b}=O(1)$, then (20) is valid as a leading order approximation and agrees with the limit $d / h \gg 1$ of equation (9) in [5]. 


\section{Travelling Wave Solutions}

In order to reduce the number of parameters appearing in the governing equation, for a given wave amplitude scaling $\delta$, we choose the wavelength scale

$$
\epsilon=\sqrt{\frac{\delta}{\left|\tau-\frac{1}{3}\right|}}
$$

so that the third-derivative dispersion term becomes $O(1)$. The governing equation (11) then becomes

$$
2 \eta_{t}+3 \eta \eta_{x}=a \eta_{x x x}-b \eta^{(v)}+p \mathcal{P}_{x}[\eta]
$$

with parameters

$$
a=\left\{\begin{array}{rl}
1 & \tau>\frac{1}{3} \\
-1 & \tau<\frac{1}{3}
\end{array} \quad b=\frac{\delta}{45\left|\tau-\frac{1}{3}\right|^{2}}, \quad p=\frac{E_{b}}{\delta^{1 / 2}\left|\tau-\frac{1}{3}\right|^{1 / 2}} .\right.
$$

It should be noted that $\epsilon$ is defined in this way merely to simplify the form of the equation. If the coefficients $b$ and $p$ are large, then these terms will dictate the actual wavelength of the disturbance. For a given initial condition $\eta(x, 0)=\eta_{0}(x),(21)$ is best solved numerically using a pseudospectral scheme. Each of the terms on the right hand side are evaluated in Fourier space using fast Fourier transforms. For the nonlinear term, $\eta_{x}$ is evaluated in Fourier space but $\eta \eta_{x}$ is expressed as a product in physical space. However, here we restrict ourselves to travelling wave solutions, and in particular we are interested in the parameter regimes for which solitary travelling waves exist.

We consider travelling wave solutions in the form $\eta=2 c N(Z)$, with $Z=\sqrt{2|c|}(x-c t)$. In terms of the original dimensional variables we have

$$
\eta_{*}=2 c \delta h N(Z), \quad Z=\sqrt{2|c|} \frac{\epsilon}{h}\left(x_{*}-\sqrt{g h}(1+c \delta) t_{*}\right) .
$$

The Froude number $F$ is related to the perturbation, $\delta c$, in the propagation speed, through $F^{2}=1+c \delta$, and hence the disturbance amplitude is characterised by $\delta|c|=\left|F^{2}-1\right|$.

The equation for the travelling wave then becomes

$$
-\gamma\left(N-\frac{3}{2} N^{2}\right)=a N^{\prime \prime}-B N^{\prime \prime \prime \prime}+C \mathcal{P}(N),
$$


where the parameters are given by

$$
\gamma=\left\{\begin{array}{rl}
1 & F>1 \\
-1 & F<1
\end{array} \quad B=\frac{2\left|F^{2}-1\right|}{45\left|\tau-\frac{1}{3}\right|^{2}}, \quad C=\frac{E_{b}}{\left|2\left(F^{2}-1\right)\left(\tau-\frac{1}{3}\right)\right|^{1 / 2}},\right.
$$

with $B$ and $C$ greater than or equal to zero, from the definition of $E_{b}$. Thus for a prescribed form of electric forcing (ie $\mathcal{P}[\eta]$ given), the travelling waves are defined by the set of non-dimensional parameters $\tau, E_{b}, F$. Numerical solutions in terms of these parameters are presented later in this section, but first we seek the condition for existence of solitary waves in terms of these parameters. However, it proves simplest to first analyse the behaviour of the solution in terms of the set of variables $a, \gamma, B$ and $C$.

In the limit $B=C=0$ this equation has the soliton solution $F(Z)=$ $\operatorname{sech}^{2}(Z / 2)$ if $a \gamma<0$, but has no solitary wave solutions for $a \gamma>0$. For nonzero $B, C$ we consider the existence of true solitary waves by investigating the possibility of oscillatory solutions in the small-amplitude tail. Writing $N(Z)=e^{i k Z}$, and substituting into the linearised form of (22) gives

$$
B k^{4}+a k^{2}-C f(k)-\gamma=0,
$$

where $f(k)$ is defined in (19) for the two forms of electric field considered. For case 2 when $f(k)=k \operatorname{coth}(k D)$, there is little prospect of any progress analytically and the problem becomes numeric, so for the remainder of this paper we focus on the case when the electrode separation is much longer than the disturbance wavelength and $f(k)=|k|$. Since (24) is even in $k$ we define

$$
G(k) \equiv B k^{4}+a k^{2}-C k-\gamma,
$$

and non-decaying oscillations occur in the tail of the disturbance if $G(k)=0$ has a positive, real root with $B, C \geq 0$. Otherwise solitary wave solutions are possible.

First the case $B=0$ is considered which corresponds to dropping the fifth derivative dispersive term. Solving the quadratic for $k$ shows that if $a \gamma>0$ positive real roots for $k$ exist and so solitary waves are not possible. The only cases where there are no positive real roots for $k$ are when $a=-1, \gamma=1$ for all $C \geq 0$, and when $a=1, \gamma=-1$ with $C \leq 2$. Thus solitary wave solutions exist for $F>1, \tau<\frac{1}{3}$ for all $E_{b}$, and for $F<1, \tau>\frac{1}{3}$, if

$$
E_{b}<2\left(2\left(1-F^{2}\right)\left(\tau-\frac{1}{3}\right)\right)^{1 / 2} \text {. }
$$


For $B>0$ more careful analysis is required. Taking $F>1$ so $\gamma=1$, we see that $G(0)=-1$ and $G(k) \rightarrow \infty$ as $k \rightarrow \infty$, and hence there is at least one positive root for $k$, and an oscillatory tail appears. Thus there is no true solitary wave possible for the model equation, with $F>1$.

For $F<1$ so $\gamma=-1$, it is clear that as $C \rightarrow 0 G(k)>0$ for all $k$ and solitary waves exist, whereas when $C \rightarrow \infty$, there is a range of $k$ for which $G(k)<0$. The condition on $B, C$ dividing the two cases is that there is a double root of $G(k)=0$ with $k>0$. First considering the case $\tau>\frac{1}{3}$, solving $G(k)=G^{\prime}(k)=0$ gives a positive root, $k=k_{1}(B)$, with

$$
k_{1}^{2}=\frac{\sqrt{1+12 B}-1}{6 B},
$$

and $C=C_{*}^{(1)}(B)$ where

$$
C_{*}^{(1)}(B)=\frac{B k_{1}^{4}-k_{1}^{2}+1}{k_{1}}=\frac{1}{3} \sqrt{\frac{2}{3 B}} \frac{(12 B-1+\sqrt{1+12 B})}{(\sqrt{1+12 B}-1)^{1 / 2}} .
$$

Thus the condition that solitary waves exist for $\tau>\frac{1}{3}$ is that $C<C_{*}^{(1)}(B)$. Here $C_{*}^{(1)}(B)$ is an increasing function of $B>0$, with $C_{*}^{(1)}(0)=2$ and the region $C<C_{*}^{(1)}(B)$ is illustrated in figure $3(\mathrm{a})$.

Similar analysis shows that solitary waves exist for $\tau<\frac{1}{3}$ only if $B>\frac{1}{4}$ and $C<C_{*}^{(2)}(B)$, where

$$
C_{*}^{(2)}(B)=\frac{1}{3} \sqrt{\frac{2}{3 B}} \frac{(12 B-1-\sqrt{1+12 B})}{(\sqrt{1+12 B}+1)^{1 / 2}} .
$$

Here $C_{*}^{(2)}\left(\frac{1}{4}\right)=0$ and $C_{*}^{(2)}(B)$ is an increasing function of $B$ for $B>\frac{1}{4}$ The region of the $(B, C)$ plane in which solitary wave solutions exist for $\tau<\frac{1}{3}$ is illustrated in figure $3(\mathrm{~b})$.

In $\S 6$ the regions in which solitary wave solutions exist is expressed in terms of the dimensionless parameters $\tau$ and $E_{b}$, but before that we consider numerical solutions of the travelling wave equation (22) for sample values of the parameters $B$ and $C$, and verify the conditions (27) and (28).

\section{Numerical Solutions of Travelling Wave Equation}

In this section we obtain solitary wave solutions of (22) by considering periodic waves in the long wavelength limit using spectral methods. Assuming that the solitary wave is restricted to the region $-M \pi<Z<M \pi$, with 


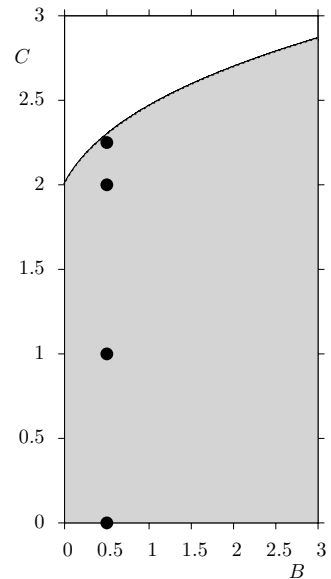

(a) $\tau>\frac{1}{3}$

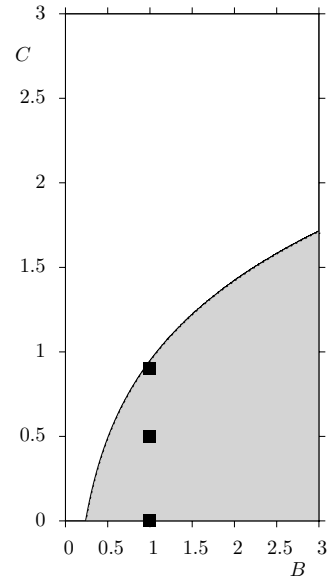

(b) $\tau<\frac{1}{3}$

Figure 3: Plot of (a) $C_{*}^{(1)}(B)$ given by $(27)$ and $(\mathrm{b}) C_{*}^{(2)}(B)$ given by (28). The shaded area identifies the region $(B, C)$ plane in which solitary wave solutions exist for $\tau>\frac{1}{3}$ and $\tau<\frac{1}{3}$ respectively. The filled circles and squares correspond to parameter values for the waveforms plotted in figures 4 and 5 respectively.

$M \gg 1$, we define $z=Z / M$, so

$$
N-\frac{3}{2} N^{2}=-\gamma\left(\frac{a}{M^{2}} N_{z z}+C \mathcal{P}[N]-\frac{B}{M^{4}} N_{z z z z}\right),
$$

and solve for $-\pi<z<\pi$.

In order to validate the predictions of $\S 4$ on the existence of solitary wave solutions, we now focus on case 1, when the electrode separation is large compared with the disturbance wavelength. Writing the waveform as a truncated Fourier sum, $N(z)=\sum_{r=-n}^{n} c_{r} e^{i r z}$, and recalling that $\mathcal{H}\left(i r e^{i r z}\right)=$ $|r| e^{i r z}$, as noted earlier, we obtain the set of nonlinear equations

$$
c_{r}-\frac{3}{2} \sum_{s} c_{r-s} c_{s}=\gamma\left(\frac{a r^{2}}{M^{2}}-\frac{C|r|}{M}+\frac{B r^{4}}{M^{4}}\right) c_{r}, \quad r=-n . . n,
$$

with unknowns $c_{r}$. Attention is restricted to real symmetric $N(z)$ in which case $c_{r}=c_{-r}$ and the set of $n+1$ equations in $n+1$ unknowns is solved using Newton's method to iterate from an initial guess. All results illustrated in this section are obtained with $N=256$ and $M=20$, though results are illustrated for a narrower range of $Z$ values for purposes of clarity. 


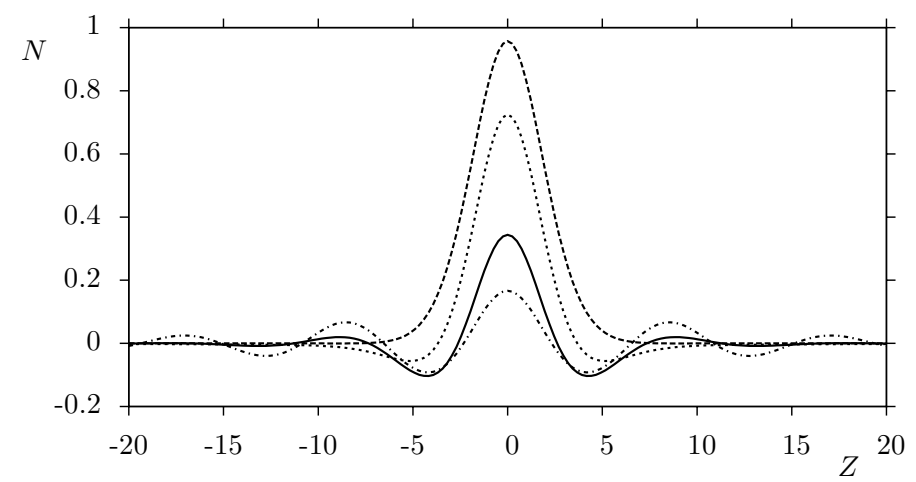

Figure 4: Wave forms for $a=1, B=0.5$ and $C=0.0,1.0,2.0,2.25$. As $C$ increases, the solution amplitude decreases and the width increases. For these parameter values, (27) predicts that travelling wave solutions exist for $C<2.29$.

We begin by considering solutions with $\gamma=-1, a=1$ (corresponding to $F<1, \tau>\frac{1}{3}$ ), where the predicted region for the existence of solitary wave solutions is illustrated in figure 3 a. Since $B=C=0$ with solution $N(z)=\operatorname{sech}^{2}(M z)$ is part of the region, this can be taken as a starting solution, with solutions for non-zero $B$ and $C$ obtained by changing the parameters by small increments starting from $B=C=0$ and using the solution for the previous values of the parameters as the initial guess. Results for $B=0.5$ and $C=0.0,1.0,2.0,2.25$ are given in figure 4 . For this particular value of $B, C_{*}^{(1)}=2.29$ and it is seen that as $C$ approaches $C_{*}^{(1)}$ the maximum wave amplitude decreases accompanied by oscillations which decay ever more slowly.

For $\tau<\frac{1}{3}$ when $a=-1$, there are no travelling waves for $B<\frac{1}{4}$, and so travelling wave forms can not be immediately generated by increasing $B$ in small increments from the $\operatorname{sech}^{2}$ profile as previously discussed. However using $N(z)=\operatorname{sech}^{2}(M z)$ as the initial guess, Newton's method converges for $B>\frac{1}{4}, C=0$. The value of $C$ can then be increased in small increments as earlier. Results for $B=1.0$ are illustrated in figure 5 . For this value of $B$ it is predicted that solitary waves exist for $C<C_{*}^{(2)}=0.93$ and results for $C=0.0,0.5,0.9$ show similar behaviour to the results shown in figure 4 in that the amplitude decreases as $C$ increases and slowly decaying oscillations arise as $C_{*}^{(2)}$ is approached.

In this section results have been presented in terms of the parameters 


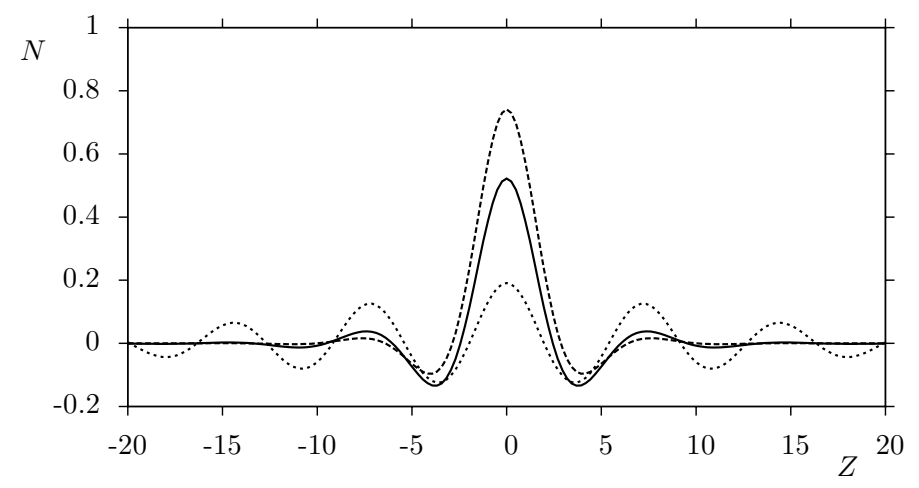

Figure 5: Wave forms for $a=-1 B=1.0$ and $C=0.0,0.5,0.9$. As $C$ increases, the solution amplitude decreases and the width increases. For these parameter values, (28) predicts that travelling wave solutions exist for $C<0.93$.

$B$ and $C$ as this is the minimum set of parameters required. In the final section the condition for the existence of solitary waves is recast in terms of the physical dimensionless parameters, $E_{b}, \tau$ and $F$.

\section{Summary}

The governing equation (11) for small-amplitude, long-wavelength disturbances has been derived in the presence of a vertical electric field due to parallel electrodes. Two cases were considered, one in which the electrode separation was long compared with the disturbance wavelength and the second case where the electrode separation is comparable to the wavelength. In the first case the analysis can be compared with [8]. The present analysis differs in that no specific relationship between wavelength and amplitude is assumed. The more general governing equation (11) agrees with the final results of the earlier work for the distinguished scalings considered there, but importantly allows an investigation of the conditions for which solitary travelling waves exist.

We conclude this paper by determining the conditions under which solitary travelling waves exist, in terms of the original non-dimensional variables $E_{b}$ and $\tau$. When the electrode separation distance is large compared with the wavelength, from $\S 4$ the condition that a solitary wave solution exists 
becomes $F<1$ and $E_{b}<E_{*}(F, \tau)$ where

$$
\begin{aligned}
E_{*}(F, \tau) & =\left(2\left(1-F^{2}\right)\left|\tau-\frac{1}{3}\right|\right)^{1 / 2} C_{*}\left(\frac{2\left(1-F^{2}\right)}{45\left|\tau-\frac{1}{3}\right|^{2}}\right), \\
C_{*}(B) & = \begin{cases}C_{*}^{(1)}(B) & \tau>\frac{1}{3}, \\
C_{*}^{(2)}(B) & \tau<\frac{1}{3} .\end{cases}
\end{aligned}
$$

Note that as $B \rightarrow \infty$, both $C_{*}^{(1)}(B)$ and $C_{*}^{(2)}(B)$ tend to $4(B / 27)^{1 / 4}$ and hence $E_{*}$ is continuous at $\tau=\frac{1}{3}$ with

$$
E_{*}\left(F, \frac{1}{3}\right)=\frac{4}{3}\left(\frac{8}{15}\right)^{1 / 4}\left(1-F^{2}\right)^{3 / 4} \text {. }
$$

Since $E_{b} \geq 0$ by definition, and $C_{*}^{(2)}(B)<0$ for $B<\frac{1}{4}$ we see that there are no solitary wave solutions for

$$
\tau<\frac{1}{3}-\sqrt{\frac{8\left(1-F^{2}\right)}{45}}
$$

The region of the $\left(\tau, E_{b}\right)$ plane for which (30) predicts the existence of solitary travelling waves with $F<1$ is shown as the shaded region in figure 6 for $F=0.9$.

These results can be compared with the case when the fifth derivative term in (21) is ignored. From (26) the condition for solitary waves solutions when $F<1$ is then $\tau>\frac{1}{3}$ and

$$
E_{b}<E_{\dagger}(F, \tau)=2\left(2\left(1-F^{2}\right)\left(\tau-\frac{1}{3}\right)\right)^{1 / 2} .
$$

This region is marked by the darker shading in figure 6 for $F=0.9$. It is seen that the conditions for the existence of solitary travelling waves agree away from the region $\tau$ close to $\frac{1}{3}$, but that the inclusion of the fifth derivative term extends the region for which solitary wave solutions exist in the neighbourhood of $\tau=\frac{1}{3}$. In particular solitary solutions are predicted with $F<1$ for values of $\tau<\frac{1}{3}$, at least when the electric field is small.

As well as providing a systematic approach to deriving wave equations for a range of different scalings and arbitrary electrode separation, the analysis 


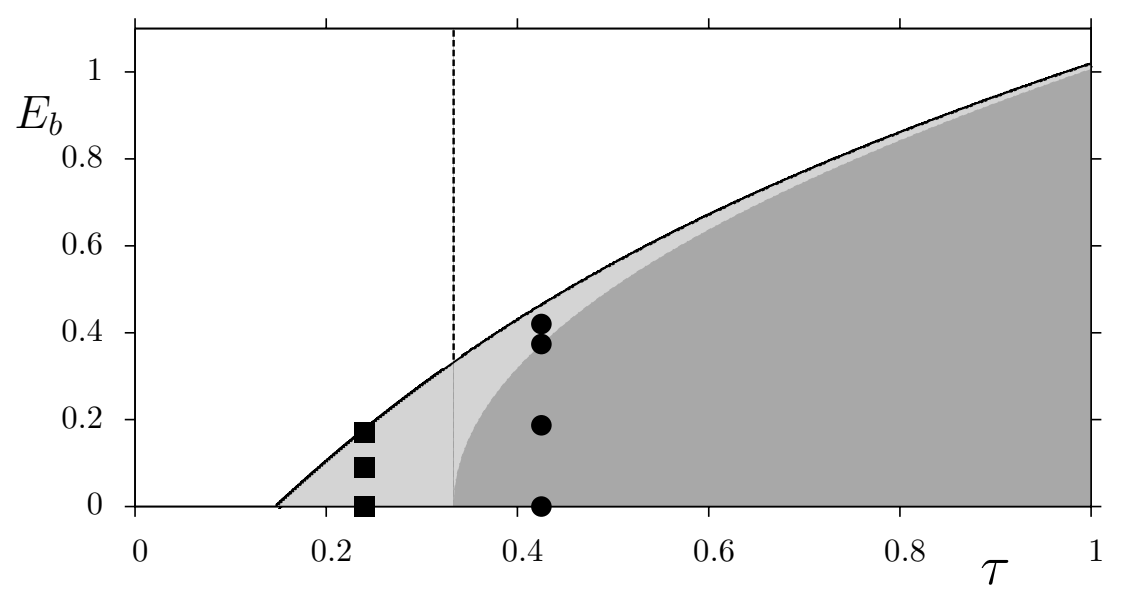

Figure 6: Region in $\tau, E_{b}$ space for which solitary wave solutions exist for $F=0.9$. The dark shaded region denotes when solitary waves exist if the fifth derivative term is ignored. The light shaded region shows the additional part of parameter space when the fifth derivative term is retained. The filled circles and squares correspond to parameter values for the waveforms plotted in figures 4 and 5 respectively. 
presented in this paper provides conditions for the existence of solitary waves in terms of the natural dimensionless parameters, Froude number $F$, electric Weber number $E_{b}$ and Bond number $\tau$. Further numerical investigation using boundary element methods for the fully nonlinear problem may clarify the validity of the weakly nonlinear approach of the present work.

\section{References}

[1] D. T. Papageorgiou, J. M. Vanden-Broeck, Antisymmetric capillary waves in electrified fluid sheets, Eur. J. Appl. Math. 15 (2004) 609-623.

[2] D. T. Papageorgiou, J. M. Vanden-Broeck, Large-amplitude capillary waves in electrified fluid sheets, J. Fluid Mech. 508 (2004) 71-88.

[3] C. V. Easwaran, Solitary waves on a conducting fluid layer, Phys. Fluids 31 (11) (1988) 3442-3443.

[4] A. Gonzalez, A. Castellanos, Korteweg-deVries-Burgers equation for surface-waves in nonideal conducting liquids, Phys. Rev. E 49 (4) (1994) $2935-2940$.

[5] A. Gonzalez, A. Castellanos, Nonlinear waves in a viscous horizontal film in the presence of an electric field, J. Electrost. 40-1 (1997) 55-60.

[6] A. Castellanos, A. Gonzalez, Nonlinear electrohydrodynamics of free surfaces, IEEE Trns. Dielectr. Electr. Insul. 5 (3) (1998) 334-343.

[7] D. T. Papageorgiou, P. G. Petropoulos, J. M. Vanden-Broeck, Gravity capillary waves in fluid layers under normal electric fields, Phys. Rev. E 72 (5) (2005) 051601-1-3.

[8] H. Gleeson, P. Hammerton, D. T. Papageorgiou, J. M. Vanden-Broeck, A new application of the Kortweg-de Vries Benjamin-Ono equation in interfacial electrohydrodynamics, Physics of Fluids 19 (2007) 031703-13 .

[9] J. K. Hunter, J. M. Vanden-Broeck, Solitary and periodic gravity capillary waves of finite-amplitude, J. Fluid Mech. 134 (1983) 205-219.

[10] J. Melcher, G. Taylor, Electrohydrodynamics: A Review of the Role of Interfacial Shear Stresses, Annual Reviews in Fluid Mechanics 1 (1) (1969) 111-146. 
[11] M. Abramowitz, I. A. Stegun, Handbook of Mathematical Functions, Dover, 1964.

[12] B. Fornberg, A Practical Guide to Pseudospectral Methods, Cambridge University Press, 1999. 